\title{
TEM and STEM Tomography for Thick Polymer Sample
}

\author{
T. Kaneko*, S. Motoki*, Y. Aoyama*, H. Nishioka*, Y. Ohkura*, Y. Kondo*, H. Jinnai** \\ * Electron Optics Division, JEOL Ltd., 1-2 Musashino 3-Chome Akishima Tokyo 196-8558, Japan \\ ** Department of Polymer Science and Engineering, Kyoto Institute of Technology, Matsugasaki, \\ Kyoto 606-8585, Japan
}

In the soft material science, meso-scale structures, ranging from sub-micrometer to a few micrometer, are known to control various properties of the materials, e.g., viscoelasticity, optical and mechanical properties etc. To clarify the relationship between the (molecular) structures and the macroscopic properties, it is important to directly observe the structures of thick sample with, at least, several periodicities in three-dimension (3D). Here, in this study, we used bright field transmission electron microscope (BF-TEM) and bright field scanning transmission electron microscope (BF-STEM) with long focal depth optical system in order to observe tomograms of an acrylonitrile butadiene styrene (ABS) resin with thickness being about $1 \mu \mathrm{m}$.

Figure 1 shows (a) BF-TEM and (b) BF-STEM images of the ABS resin. These images were taken with $200 \mathrm{kV}$ TEM/STEM (JEM-2100), the pixel resolution is $4 \mathrm{~nm}$ and the effective focal depth is $1.6 \mu \mathrm{m}$. The gray part of the electron micrograph corresponds to the $\mathrm{OsO}_{4}$-stained polybutadiene (PB) domain. It was found that the electron micrograph in Figure 1(a) was blur than that in Figure $1(\mathrm{~b})$, probably due to the chromatic aberration in the BF-TEM.

Figure 2 shows the 3D reconstructed images of (a) BF-TEM tomography and (b) BF-STEM tomography. $\mathrm{PB}$ and acrylonitrile/styrene (PA/PS) domains are shown in dark blue and light blue, respectively. In both images, ball-like PB domains can be observed.

The $\mathrm{x}-\mathrm{z}$ digital slices of 3D reconstruction for BF-TEM and BF-STEM tomograms at the same place of the ABS resin are shown in Figure 3. The z-axis is parallel to the optical axis of the microscope. The round shaped gray domains and white domains correspond to PB and PA/PS domains, respectively. The $x-z$ digital slice of BF-TEM tomogram (see Figure 3 (a)) seems to be blur again due to chromatic aberration. On the other hand, the spatial resolution for $\mathrm{x}-\mathrm{z}$ digital slice of BF-STEM tomogram (see Figure 3(b)) continuously decreases from top to bottom the image. Namely, the fine structures (whose size is ca. $10 \sim 15 \mathrm{~nm}$ ) inside the PB domains marked by the solid circle appeared more clearly that those marked by the dashed circle.

Goldstein et al., estimated the electron beam broadening width $(b)$ in thick samples (see Figure 4) [1]. According to the Goldstein's equation, $b$ is roughly $10 \mathrm{~nm}$, when sample thickness $(t)$ is $500 \mathrm{~nm}$, which is comparable with the size of included PA/PS domains. This is probably why the fine structures of PA/PS domains are unclear in the lower PB domains. This phenomenon implies that the electron beam broadening plays an important role for the spatial resolution of the $3 \mathrm{D}$ reconstructed tomogram.

Reference

[1] J. I. Goldstein, Introduction to Analytical Electron Microscopy, Plenum Press, New York, 1979 

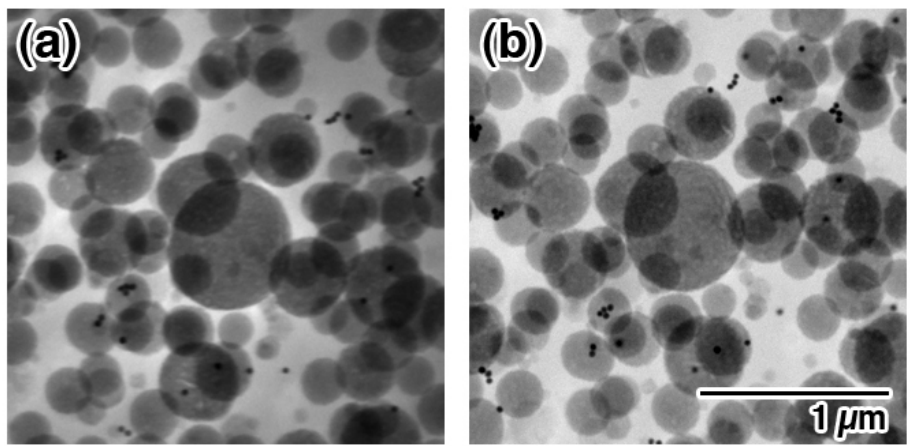

FIG. 1. The electron micrographs of (a) BF-TEM and (b) BF-STEM of an ABS resin stained by $\mathrm{OsO}_{4}$. Grey domains correspond to PB domains. The thickness of the ABS plastics was ca. $1 \mu \mathrm{m}$.

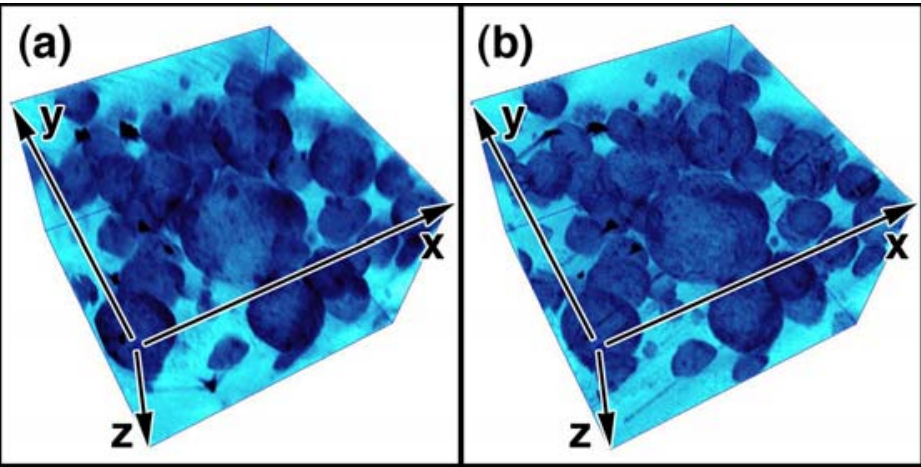

FIG. 2. 3D reconstructed tomograms of the ABS resin by (a) BF-TEM and (b) BF-STEM. Box size is $1.4 \mu \mathrm{m} \times 1.4 \mu \mathrm{m} \times 1.0 \mu \mathrm{m}$. The thick sample was tilted around the y-axis, and the optical axis of the microscope is z-axis.
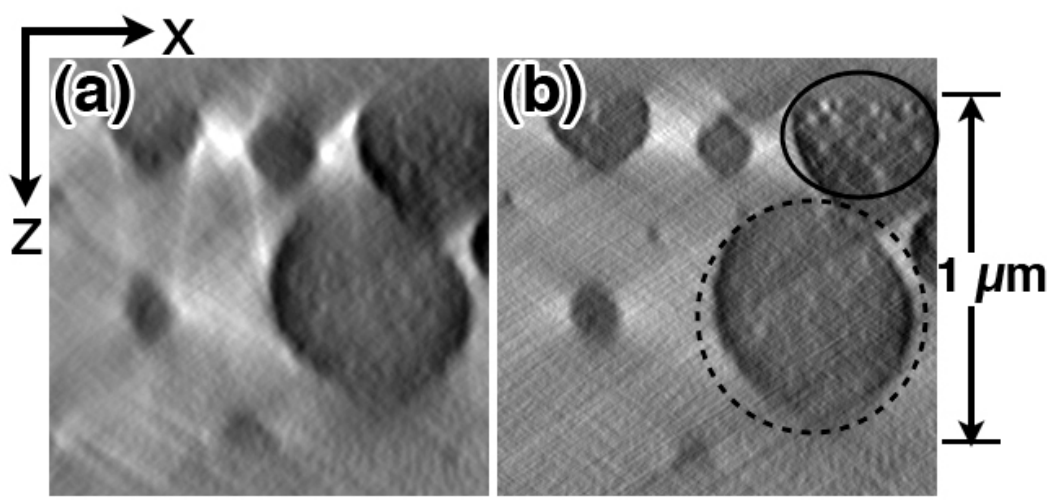

FIG. 3. The $x-z$ digital slice images of (a) BF-TEM and (b) BF-STEM tomograms. The thickness of the sample was estimated to be ca. $1 \mu \mathrm{m}$.

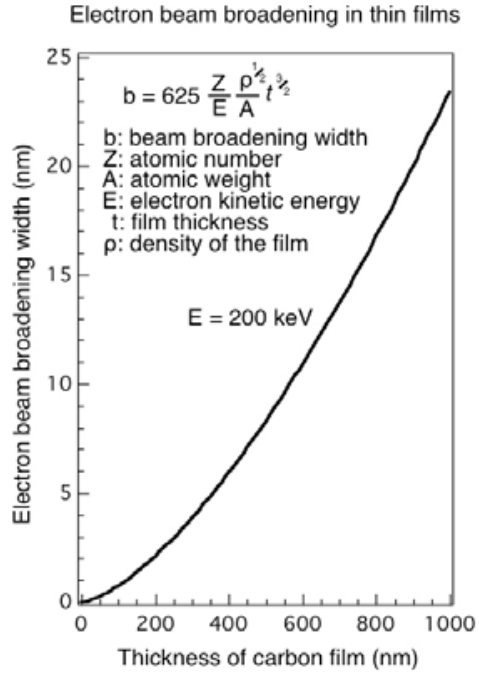

FIGS. 4. Plot of the electron beam broadening width versus thickness of carbon film. Goldstein's equation was shown in the inset. 